\section{Nucleoporin-mediated regulation of cell identity genes}

\author{
Arkaitz Ibarra, ${ }^{1}$ Chris Benner, ${ }^{2,3}$ Swati Tyagi, ${ }^{1}$ \\ Jonah Cool, ${ }^{1,4}$ and Martin W. Hetzer ${ }^{1}$
}

${ }^{1}$ Molecular and Cell Biology Laboratory, ${ }^{2}$ Integrative Genomics
and Bioinformatics Core, Salk Institute for Biological Studies, La
Jolla, California 92037, USA

The organization of the genome in the three-dimensional space of the nucleus is coupled with cell type-specific gene expression. However, how nuclear architecture influences transcription that governs cell identity remains unknown. Here, we show that nuclear pore complex (NPC) components Nup93 and Nup153 bind superenhancers $(\mathrm{SE})$, regulatory structures that drive the expression of key genes that specify cell identity. We found that nucleoporin-associated SEs localize preferentially to the nuclear periphery, and absence of Nup153 and Nup93 results in dramatic transcriptional changes of SEassociated genes. Our results reveal a crucial role of NPC components in the regulation of cell type-specifying genes and highlight nuclear architecture as a regulatory layer of genome functions in cell fate.

Supplemental material is available for this article.

Received July 17, 2016; revised version accepted October 7, 2016.

Cell type-specific gene expression relies on the accurate spatial and temporal control of transcription. Thousands of enhancers contribute to the execution of specific gene expression programs. The activity of key genes crucial for cell type identity is driven by a small fraction of regulatory elements composed of clusters of enhancers, referred to as stretch enhancers or superenhancers (SEs) (Hnisz et al. 2013; Parker et al. 2013). The function of enhancers is influenced by the topological organization of the genome, which is emerging as a major regulator in the maintenance of cell type-specific gene expression (Cavalli and Misteli 2013; Heinz et al. 2015). While the precise mechanisms underlying genome organization remain under scrutiny, large-scale spatial chromosome architecture partially relies on interactions with the nuclear envelope (NE) (Mekhail and Moazed 2010; Van de Vosse et al. 2011). Components of the NE and nuclear pore complexes (NPCs) associate with the genome to influence its organization and gene expression (Van de Vosse et al. 2011). However, the potential contribution of NE components in the regulation of cell type-specific transcriptional programs is still poorly understood.

[Keywords: nuclear architecture; nucleoporin; nuclear pore complex; cell identity; superenhancer; transcription]

Present addresses: ${ }^{3}$ Department of Medicine, University of California at San Diego, San Diego, La Jolla, CA 92093, USA; ${ }^{4}$ Organovo Holdings, Inc., San Diego, CA 92121 , USA.

Corresponding author: hetzer@salk.edu

Article published online ahead of print. Article and publication date are online at http://www.genesdev.org/cgi/doi/10.1101/gad.287417.116.
The double-lipid bilayer of the NE is composed of the outer nuclear membrane (ONM) and inner nuclear membrane (INM) and is perforated at multiple sites by NPCs. High-resolution images of the nuclear periphery of several human cell types show that the NE is mostly underlined with heterochromatin, except at the NPCs /Capelson and Hetzer 2009). Consistent with these observations, the lamins, a meshwork of intermediate filaments that underlie the NE, interact with large transcriptionally repressed genomic regions at the nuclear periphery, the lamina-associated domains (LADs) (Guelen et al. 2008). In contrast, genomic regions at NPCs appear largely devoid of heterochromatin in human cells (Capelson and Hetzer 2009). Each NPC is composed of several copies of $\sim 30$ different subunits, called nucleoporins (Nups). Structurally, the NPC is divided into a NE-embedded scaffold, comprised of the Nup93-Nup205 and Nup96-Nup107 subcomplexes, and two peripheral structures, the cytoplasmic filaments and the nuclear basket. The latter extends into the nuclear interior and is composed of Nup153 and Tpr (D'Angelo and Hetzer 2008). In addition to the well-characterized role of NPCs in the regulation of nucleocytoplasmic transport of macromolecules, studies mainly in yeast and Drosophila revealed that NPC components associate with specific genomic regions to positively or negatively regulate the transcriptional activity of target genes (for review, see Ibarra and Hetzer 2015). While most Nupgenome interactions in yeast occur at the NPC, in metazoans, dynamic Nups can also extensively interact with the genome away from the NPC /Capelson et al. 2010; Kalverda et al. 2010). In human cells, characterization of Nup-genome interactions is still in its infancy (Brown et al. 2008); nevertheless, the relevance of Nups in the regulation of genome functions has been inferred from the mutations of NPC components that lead to a panel of tissue-specific pathologies (Capelson and Hetzer 2009; Nofrini et al. 2016). It has been speculated that Nup functions in gene expression regulation might underlie their involvement in tissue-specific disorders (Raices and D'Angelo 2012). However, whether Nups could recognize and regulate the expression of cell type-specific genes in human cells remains unknown.

\section{Results and Discussion}

\section{NPC and INM components interact with functionally opposite chromatin regions at the NE}

To gain insights into the role of NE proteins in the regulation of genome functions in human cells, we used the DamID (DNA adenine methyltransferase identification) technique (Guelen et al. 2008; Kalverda et al. 2010; Meuleman et al. 2013; Jacinto et al. 2015) to identify interactions between NE components and the genome. We first validated this approach by generating genome interaction maps in U2OS cells for the Lamin B receptor (LBR), an INM protein that interacts with Lamin B and contributes

(C) 2016 Ibarra et al. This article is distributed exclusively by Cold Spring Harbor Laboratory Press for the first six months after the full-issue publication date (see http://genesdev.cshlp.org/site/misc/terms.xhtml). After six months, it is available under a Creative Commons License (Attribution-NonCommercial 4.0 International), as described at http://creativecommons.org/licenses/by-nc/4.0/. 
to the localization of heterochromatin to the nuclear periphery in differentiated cells (Solovei et al. 2013). We generated stable cell lines expressing the Dam fused to LBR or GFP as a reference. We found that LBR associated with 1046 large chromosome segments with a median size of 766 kb (Fig. 1A; Supplemental Fig. S1A). LBR-associated regions effectively recapitulate the Lamin B1 DamID interaction maps described in HT1080 cells (Meuleman et al. 2013) and Tig3 cells (97\% and 79\% overlap, respectively) (Fig. 1A; Guelen et al. 2008). This indicates that, as expected, LBR associates with the LADs, supporting a robust conservation of genome contacts with the nuclear lamina (Meuleman et al. 2013). Next, to expand our understanding of NE-genome interactions, we performed similar experiments with components of the NPCs, such as the nuclear basket member Nup153 and the scaffold constituent Nup93. Using a Dam-GFPexpressing cell line to correct for the effects of local chromatin accessibility (Guelen et al. 2008), we identified 1851 local genome interaction sites with the basket component Nup153 (Fig. 1B,I). In addition, we detected 1021 interaction sites for the more secluded NPC component Nup93 that accumulate in very close proximity to Nup153-binding sites (Fig. 1B; Supplemental Fig. S1B), likely representing bona fide interactions with the NPC.

We next characterized the genomic, transcriptional (mRNA sequencing [RNA-seq]), and chromatin (chromatin immunoprecipitation [ChIP] combined with highthroughput sequencing [ChIP-seq] of epigenetic modifica- tions and DNase I hypersensitivity site [DHS] maps) features of LBR, Nup153, and Nup93 interactions with the genome. LBR-associated domains presented lower gene density, low mRNA expression levels, low chromatin accessibility (DHSs), lower occupancy of the insulator protein $\mathrm{CTCF}$, and low levels of the central histone modifications found at active promoters (H3K4me3) and regulatory elements (H3K27ac) (Fig. 1C-E; Supplemental Fig. S1C-F). Instead, LBR-associated domains exhibited higher levels of the repressive histone modification H3K9me2 (Fig. 1F). In contrast, Nup153 and Nup93 interaction sites accumulated closer to transcription start sites than LBR LADs (Supplemental Fig. S1G), mostly in promoter-distal intergenic $(\sim 53 \%$ of Nup153 and $51 \%$ of Nup93 interactions) and intragenic ( $39 \%$ of Nup153 and $40 \%$ of Nup93 interactions) regions (Fig. 1I; Supplemental Fig. S1H). Nup153 and Nup93 interaction sites were enriched for histone modifications associated with active transcription such as $\mathrm{H} 3 \mathrm{~K} 4 \mathrm{me} 3$ and, predominantly, H3K27ac, which marks putative enhancers (e.g., up to $\sim 50.5 \%$ of Nup153 interaction sites occur at H3K27ac regions; $P<1 \times 10^{-10}$ ) (Fig. 1J; Supplemental Fig. S1I). Interaction sites of these Nups also correlated with DHSs, which characterize active regulatory regions (Fig. 1J; Supplemental Fig. S1I; Song and Crawford 2010). Furthermore, the top de novo motif identified within Nup153-binding sites was the regulatory DNA sequence AP1, supporting the idea that Nup153 interacts with genomic regulatory regions, including putative transcriptional enhancers (Supplemental Fig. S1J).

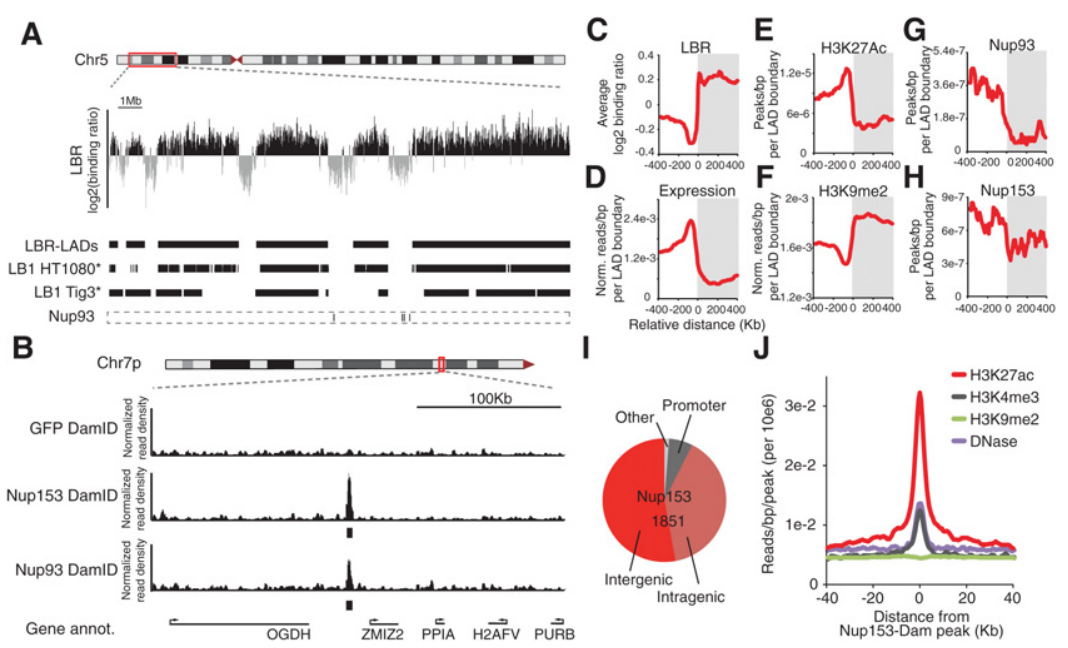

Figure 1. NPC components and LBR interact with functionally opposite chromatin compartments. (A) LBR-binding profile in U2OS cells ( $\log _{2}$ ratio of Dam-LBR to Dam-GFP methylation). The interaction profile along a region of chromosome 5 is shown. Black rectangles indicate LADs identified using LBR-DamID replicates in U2OS cells or Lamin B1 (LB1) DamID data in Tig3 cells (Guelen et al. 2008) and HT1080 cells (Meuleman et al. 2013). Nup93 DamID interaction sites are shown in the box. (B) DamID interaction profiles of GFP, Nup153, and Nup93 in a segment of chromosome 7. High-confidence interaction sites are indicated with black tracks. $(C-H)$ LBR-defined LADs were aligned by their boundaries, and the occupancy of LBR $(C)$, mRNA production $(D)$, H3K27ac $(E), \mathrm{H} 3 \mathrm{~K} 9 \mathrm{me} 2$ $(F)$, Nup93 $(G)$, and Nup153 $(H)$ was examined. Gray areas highlight LADs. Lines show moving-window averages of $25 \mathrm{~kb}$ in size. (I) Distribution of Nup153 interaction sites obtained from two biological replicates relative to annotated genes. Interactions with promoters $( \pm 1 \mathrm{~kb}$ from the transcription start site [TSS]), intragenic regions (exons and introns), intergenic regions, and other features are shown as indicated. (J) Histogram showing the distribution of chromatin features (H3K27ac in red, H3K4me3 in gray, DNase I hypersensitivity sites [DHSs] in purple, and $\mathrm{H} 3 \mathrm{~K} 9 \mathrm{me} 2$ in green) relative to Nup153 interaction sites.

\section{Nups interact with SES}

When we examined the distribution of NEgenome interactions, Nup93 and Nup153 interactions were found to accumulate outside of LBR-defined LADs (Fig. 1A,G,H) and gather around large clusters of transcriptional enhancers, such as the one located near the Myc oncogene (Fig. 2A; Supplemental Fig. S2A-D; Hnisz et al. 2013). Key cell type-specifying genes are generally regulated by SEs (Hnisz et al. 2013; Parker et al. 2013), which can be defined by characteristically high levels of H3K27ac (Hnisz et al. 2013). To evaluate the presence of these Nups at SEs, we identified the collection of SEs in U2OS cells defined by H3K27ac (345 SEs out of 11,831 putative enhancers) (Fig. 2B; Hnisz et al. 2013). Surprisingly, Nup153 interactions sites were enriched in SEs $\left(P<<1 \times 10^{-10}\right)$ and associated with 174 of 345 SEs (50.4\%) (Fig. 2D). Nup93 interaction sites were also enriched in SEs $\left(P<<1 \times 10^{-10}\right) ;$ of 345 SEs, $104(30.1 \%)$ showed association with Nup93, $83.6 \%$ of which were bound by both NPC components (Supplemental Fig. S2A-F).

In U2OS osteosarcoma cells, SE-associated genes are involved in bone physiology and hallmarks of cancer (e.g., Myc and regulation of cell cycle) (Fig. 2A-C; Hnisz et al. 2013). The subset of Nup-bound SEassociated genes participates in similar 
A

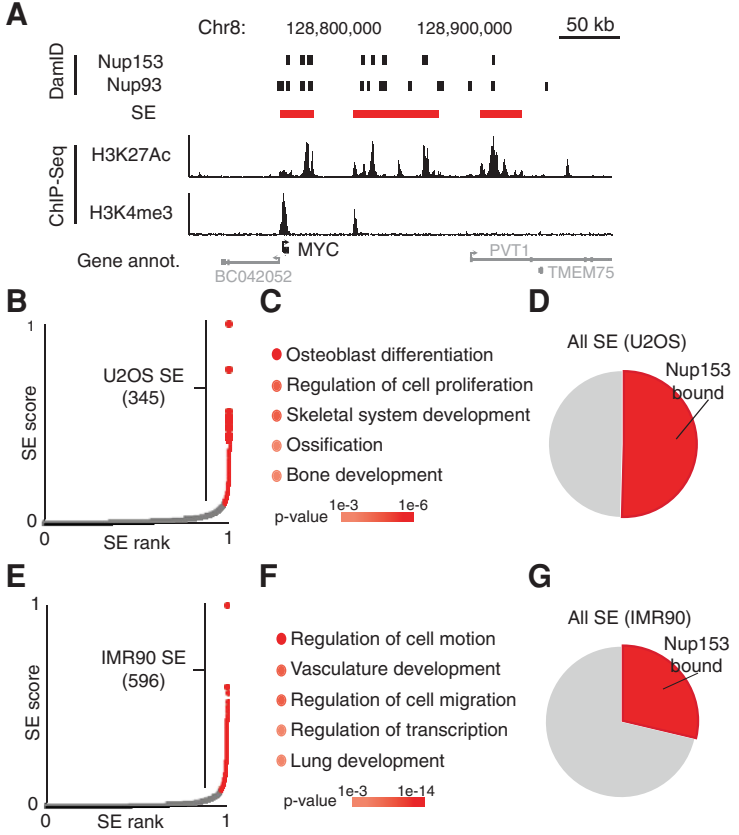

Figure 2. NPC components Nup153 and Nup93 interact with SEs in different cell types. (A) Interaction sites of Nup153 and Nup93 at the regulatory region surrounding the $M y c$ gene are depicted with black bars, and SEs are indicated with red bars. Normalized $\mathrm{H} 3 \mathrm{~K} 27 \mathrm{ac}$ and $\mathrm{H} 3 \mathrm{~K} 4 \mathrm{me} 3$ profiles and University of California at Santa Cruz (UCSC) genes illustrate the chromatin and genomic landscape. $(B, E)$ Enhancer regions ranked by H3K27ac levels in U2OS $(B)$ and IMR90 $(E)$ cells. SEs are highlighted in red (see the Supplemental Material). $(C, F)$ Selected gene ontology categories of SE-associated genes in U2OS $(C)$ and IMR90 $(F)$ cells. $(D, G)$ Pie charts showing the fraction of SEs that interact with Nup153 (red) in U2OS $(E)$ and IMR90 $(G)$ cells.

biological processes (Supplemental Fig. S2G), including genes critical for ossification (Ext1) (Koziel et al. 2004) and osteoblast compaction (Itgb1) (Brunner et al. 2011) among others (Fig. 4C; Supplemental Fig. S2G,H).

To confirm the association between SEs and Nups, we explored this link in an unrelated cell type; namely, IMR90 primary lung fibroblasts. Based on H3K27ac levels, we identified 596 SEs in IMR90 cells (out of 17,746 putative enhancers) (Fig. 2E), which were associated with genes involved in cell migration, lung development, and the regulation of transcription among other processes (Fig. 2F). Next, since we detected a stronger relationship between SEs and Nup153, we performed DamID and sorted the top 1851 Nup153-binding sites throughout the IMR90 genome to operate with a similar number of interactions in both cell lines. We observed a consistent enrichment of Nup153 at IMR90 SEs (171 of 596 SEs; $P<<1 \times 10^{-10}$ ) (Fig. 2G), which were associated with key genes involved in lung development (Smad7) and cell adhesion (Bcar1 and Ltbp2) (Nojima et al. 1996; Hyytiäinen and Keski-Oja 2003) among others (Supplemental Fig. S3A-E).

To test whether Nup-SE interactions exhibited cell type specificity, we classified the SEs into U2OS-specific, IMR90-specific, and "common" SEs overlapping in both cell lines (Supplemental Fig. S3F). We then compared U2OS-specific and IMR90-specific SEs with Nup153 interaction maps identified in U2OS and IMR90 cells. Nup153 interaction sites identified in each cell type showed better overlap with the respective cell type-specific SEs (Supplemental Fig. S3G). This suggests that Nup153 preferentially interacts with cell typespecific SEs.

\section{SEs interactions with Nups are enriched at the nuclear periphery}

FRAP (fluorescence recovery after photobleaching) analyses showed that scaffold NPC components such as Nup93 are remarkably stable at the NE-embedded NPC, whereas the nuclear basket component Nup153 presents a stable and a dynamic subpopulation, the latter exchanging between the NPC and the nucleoplasm (Griffis et al. 2004; Rabut et al. 2004). To determine the subnuclear localization of SEs that interact with NPC components, we performed DNA fluorescent in situ hybridization (FISH) in U2OS and IMR90 cells (Supplemental Table S1). We determined the relative radial positions of FISH signals in the middle plane of the nucleus binned into three concentric zones of equivalent area (Fig. 3A; Towbin et al. 2012). As a reference, we used probes covering actively expressed genes and SEs that do not interact with Nups (expected to localize to the nuclear interior) as well as probes within LADs (expected to be peripheral). We then examined the positions of 19 Nup-associated SEs (11 Nup153-associated SEs in IMR90 and eight in U2OS, six of which were cobound by Nup93). We observed the preferential accumulation of these SEs in the most peripheral subdivision of the nucleus in both cell types (Fig. 3B,C; Supplemental Fig. S4A,B). In IMR90 cells, 51.9\%, 26.8\%, and $21.1 \%$ of measured Nup153-associated SE loci mapped respectively to zones 1,2 , and 3 , on average, compared with $25.2 \%, 40.3 \%$, and $34.5 \%$ of control active regions and $62.5 \%, 25.3 \%$, and $12.2 \%$ of loci within LADs. Furthermore, fractionation of the radius in 10\% windows showed that the global nuclear distribution of Nup153-associated SEs in IMR90 cells was more proximal to the NE than control active regions $(\sim 34 \%$ of all Nup-associated SE signals were detected in the outer fraction of the nucleus compared with $\sim 14 \%$ of control active regions) (Fig. 3D). Consistently, in U2OS cells, up to $49 \%$ of the Nup-bound SE foci mapped to the peripheral zone 1 on average, compared with only $24 \%$ of control active regions (Supplemental Fig. S4B). The tested SEs that exhibited association only with Nup153 in U2OS also showed peripheral localization. This suggests that the presence of this Nup directly facing the chromosomes at the nuclear basket allows the detection of additional DamID interactions compared with the secluded scaffold component Nup93. Interestingly, silenced regions embedded within LADs are the most restricted to the NE in both cell types (Fig. 3D). This suggests that, compared with LADs, NPC-associated regions show a more dynamic behavior, likely subjected to cell cycle-dependent fluctuations as reported in yeast (Brickner and Brickner 2010). Moreover, SEs were mostly excluded from LADs (Supplemental Fig. S4C), denoting that their localization is not driven by components of the nuclear lamina. Together, our results indicate that Nup153 and Nup93 interact with a subset of SEs in different cell types predominantly at the nuclear periphery, suggesting contact of these SEs with the NPCs. 


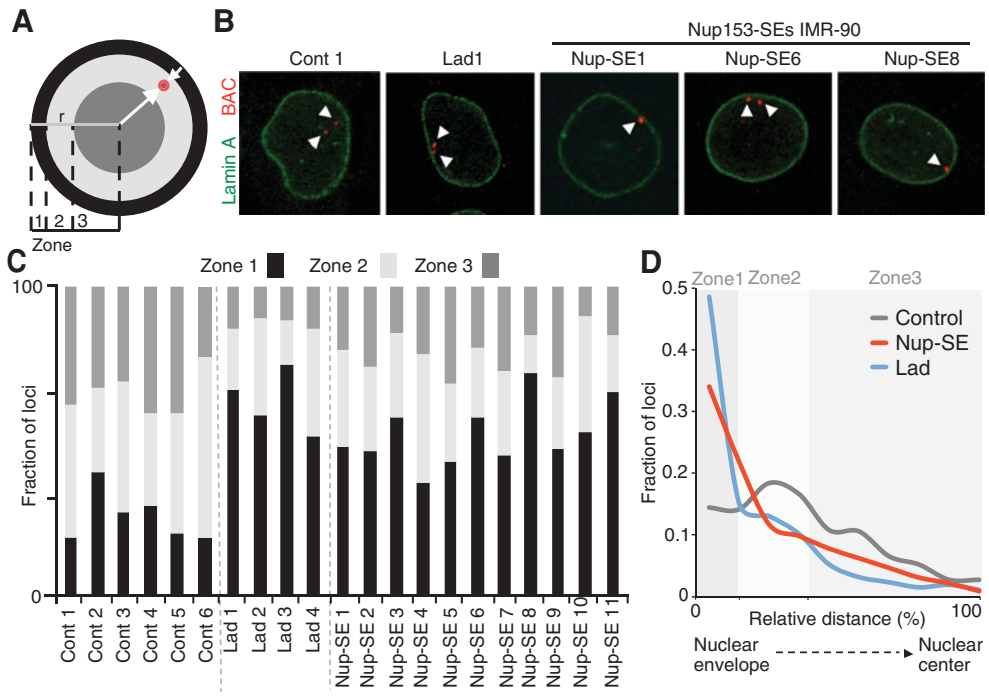

Figure 3. Subnuclear localization of Nup-associated SEs. (A) Subdivision of the central plane of the nucleus in three concentric regions of equal area: the most peripheral zone 1 (black; 0-18.4), zone 2 (light gray; 18.4-42.3), and zone 3 in the nuclear interior (dark gray; 42.3-100). (B) Representative immunofluorescence (IF) DNA-FISH images of the indicated regions in IMR90 cells. Lamin A (green) and DIG-labeled BAC probes (red) are shown. (C) Distribution of analyzed loci into the three zones, represented as in $A$. (Cont) Control active regions; (Lad) regions within LADs; (Nup-SE) Nup153-associated SEs. $n>50$ for each region. $(D)$ Distribution of the relative radial distance of all measured control regions (gray; $n=406$ ), Nup153-associated SEs (red; $n=622$ ), and LAD regions (blue; $n=240$ ). Gray shading indicates the theoretical positioning of the three zones.

Nups regulate the transcriptional activity of SE-associated genes

NPC components like Nup153 regulate gene expression in Drosophila and mouse cells without dramatically affecting global transport or nuclear compartmentalization (Supplemental Fig. S5; Vaquerizas et al. 2010; Jacinto et al. 2015). We therefore explored the potential role of NPC components in regulating the expression of SE-associated genes in human cells. In regard to Nup153, RNA-seq experiments showed that knockdown of this Nup led to global transcriptional changes in U2OS cells comparable with those reported in other organisms /false discovery rate $[$ FDR $]<0.05$ and fold change $>1.5 ; 2440$ up-regulated and 2568 down-regulated genes) (Supplemental Fig. S6A; Supplemental Table S2; Vaquerizas et al. 2010; Jacinto et al. 2015). Moreover, Nup153 interaction sites were associated with differentially expressed genes, revealing that Nup153 is required for the accurate expression of target genes in human cells (Supplemental Fig. S6B). Remarkably, expression levels of SE-associated genes were significantly more affected by Nup153 knockdown than the rest of the genes $(P<0.01)$ (Fig. 4A,B). A large subset of Nup153-associated SE-driven genes experienced robust expression changes upon Nup153 depletion (Fig. 4C; Supplemental Fig. S6D,E). Furthermore, Nup93 knockdown resulted in similar transcriptional defects of SE-associated genes, suggesting that several NPC components might cooperate to regulate the transcriptional activity of these genes (Supplemental Fig. S6C-E). Consistently, Nup153 knockdown led to gene expression changes in IMR90 cells (Supplemental Table S3), and transcriptional alterations were particularly severe for SE-associated genes compared with the rest of the genes $(P<<0.01)$ (Fig. 4D-F; Supplemental Fig. S3C,D). To further control for the high expression levels often associated with SE-associated genes, we compared the effects of Nup153 knockdown on genes with the same expression levels but not driven by SEs. SE-associated genes were more likely to be regulated by Nup153 knockdown in IMR90 and U2OS cells (Supplemental Fig. S6F). These data suggest that, in different cell types, NPC components contribute to the accurate expression of genes encoding key components that drive cell type specification.

Our results indicate that the NE acts as a platform for regulating the genome functions in human cells. In contrast to the repressive chromatin landscape at LADs, NPCs maintain the activity of a transcriptionally malleable environment. Previous attempts to map Nup-genome interactions in differentiated human cells, while incomplete, supported a notion of context-dependent association of NPC components with transcriptionally active and repressed regions (Brown et al. 2008; Liang et al. 2013). In agreement with our observations, recent studies in human $\mathrm{CD}^{+} \mathrm{T}$ cells proposed that HIV integration occurs at putative NPC interaction sites, which are transcriptionally active regions that localize at the nuclear periphery and are excluded from LADs (Marini et al. 2015). Our results are also consistent with the patterns of chromatin organization observed in differentiated human cells using high-resolution imaging techniques (Capelson and Hetzer 2009) and provide genetic and molecular details of this organization.

A subset of metazoan NPC components, the dynamic Nups, exchanges between the NPC and the nucleoplasm (Rabut et al. 2004). Consistently, Nup153 can interact with the genome both at the NPC and away from it in Drosophila and mouse cells (Vaquerizas et al. 2010; Jacinto et al. 2015). It remains unclear whether Nup-genome interactions that occur away from the NPC are functionally distinct from NPC-associated sites. Importantly, our results in human cells indicate that Nup-associated SEs show preferential localization at the nuclear periphery, where NPCs might provide large and stable platforms to accurately regulate the expression of key SE-associated genes. However, additional interactions between Nups and SEs might exist in the nuclear interior that were missed due to technical and analytical limitations. For instance, since DamID relies on very low expression levels of the Dam fusion proteins (Guelen et al. 2008), the methylation levels around the NPCs where multiple copies of Nups accumulate might be more prominent compared with the dynamic pools. Nevertheless, the peripheral localization of a subset of SEs suggests that noncanonical enhancer characteristics, in particular their association with features of the nuclear landscape, could contribute to the emerging definition of SEs (Pott and Lieb 2015).

Mutations affecting the NE and NPC components underlie a panel of tissue-specific disorders and developmental pathologies (Capelson and Hetzer 2009; Worman et al. 2010; Raices and D'Angelo 2012). In addition, NPC components have been implicated in cell differentiation and cell identity maintenance (D'Angelo et al. 2012; Buchwalter et al. 2014; Jacinto et al. 2015). Here, we show 
A
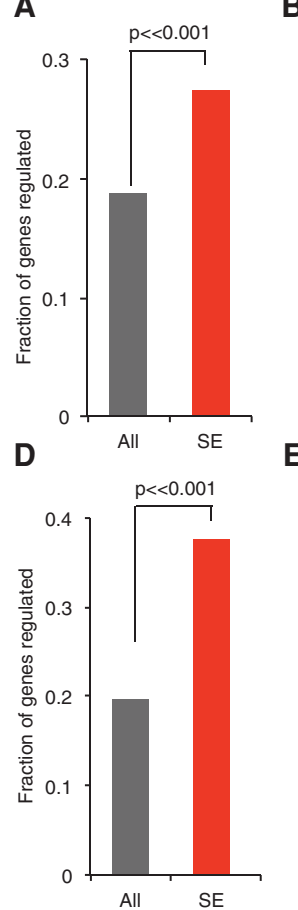

B
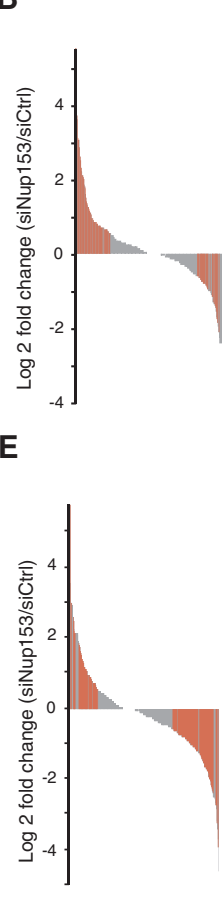

C

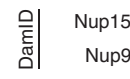

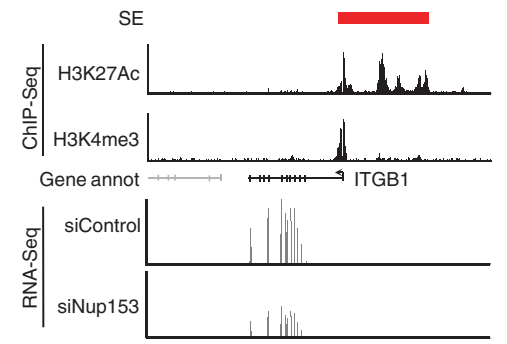

$\mathbf{F}$
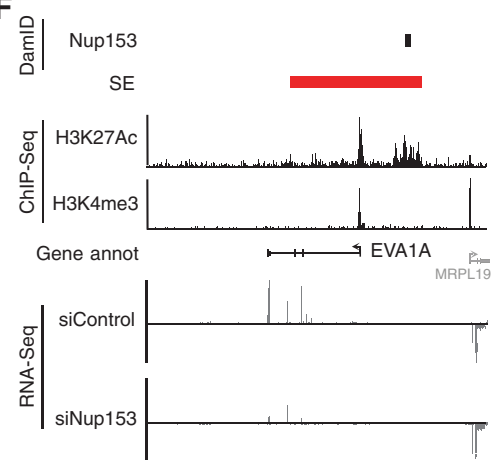

Figure 4. Nup153 regulates the accurate transcriptional activity of cell identity genes in different cell types. $(A, D)$ Bar graphs showing the regulation exerted by Nup153 over SE-associated genes (red) and the rest of the genes (gray) in U2OS $(A)$ and IMR90 $(D)$ cells. FDR $<0.05 ;>1.5$-fold differential expression. $P$-value, Fisher exact test. $(B, E)$ SE-associated genes ranked according to their changes in transcriptional activity upon Nup153 knockdown relative to control populations in U2OS $(B)$ and IMR90 $(E)$ cells. Differentially expressed genes are highlighted in red. $(C, F)$ Examples of Nup-bound SE-associated cell identity genes in U2OS $(C)$ and IMR90 $(F)$ cells. Nup153- and Nup93-binding sites are shown with black bars, and SEs are shown with red bars. UCSC genes and normalized H3K4me3, H3K27ac, and RNA-seq profiles in each cell type are shown.

that components of the NPC, such as Nup153 and Nup93, establish functional interactions with SE-associated genes, which are critical regulators of cell type-specific transcriptional programs. Thus, our results support a model in which Nup functions in gene expression regulation underlie their involvement in tissue-specific disorders and cell identity maintenance (Supplemental Fig. S7). Consistently, Nup98 interacts with lineage-specific genes in neural progenitor cells (Liang et al. 2013), and, in mouse embryonic stem cells (mESCs), Nup153 depletion results in heterogeneous gene expression changes that lead to loss of pluripotency (Jacinto et al. 2015). Interestingly, in mESCs, Nup153 regulates a subset of genes through interactions with Polycomb group proteins (PRC1), which promote predominantly gene silencing but also gene activation (Aranda et al. 2015; Jacinto et al. 2015). In addition, Nup153 has also been reported to interact with histone acetyltransferases such as p300/CBP, responsible for most H3K27 acetylation and highly enriched at SEs (Kasper et al. 1999; Vahedi et al. 2015), which could facilitate the Nup-SE interaction. Intriguingly, Polycomb and SE domains share a similar topological organization that serves to insulate these regions (Dowen et al. 2014). Since NPC components display insulator activity and regulate long-range chromatin interactions in yeast (Ishii et al. 2002; Tan-Wong et al. 2009), it is tempting to speculate that a structural role of Nups in chromosome organization might influence the accurate transcriptional activity at SE and Polycomb domains.

Together, our results highlight a key function of Nups in the regulation of cell typespecific gene expression and anticipate a prominent role for the NE components in the regulation of genome functions during cell fate specification and disease.

\section{Materials and methods}

\section{Cell culture and manipulation}

U2OS cells were cultured in DMEM (Gibco) and 10\% FBS. IMR90 cells were cultured in DMEM and 20\% FBS supplemented with nonessential amino acids (Gibco) and lowoxygen conditions $(3 \%)$. Cell lines expressing Dam fusion proteins were generated by retroviral infection and selected with $1 \mu \mathrm{g} / \mathrm{mL}$ puromycin. siRNA-mediated gene silencing was performed using the oligos (Invitrogen) control (Luc; 5'-UAUGCAGUUGCUCUCCAGC-3'), Nup153 (5'ACAUUUGGUAGAGUCUGCCUU-3'), and Nup93 (5'GCGCUAAUUUACUACUGCA-3') and were delivered using siLenFect (Bio-Rad). DNA transfections were carried out using Lipofectamine 2000 (Invitrogen) and $1 \mu \mathrm{g}$ of DNA.

\section{DamID}

Genomic DNA from stable cell populations expressing Dam fusion proteins was isolated using the DNeasy blood and tissue kit (Qiagen). DamID samples were processed and analyzed as described previously (Jacinto et al. 2015). Detailed information about sample preparation and data analysis is in the Supplemental Material.

\section{RNA-seq}

Total RNA was isolated from the different cell populations using Trizol (Ambion) and purified using the RNAeasy kit (Qiagen). Total RNA (1-2 $\mu \mathrm{g}$ ) was processed using the Illumina Tru-seq stranded RNA LT kit (Illumina) to obtain strand-specific mRNA libraries. Resulting libraries were run in a HiSeq 2500 sequencing system according to the manufacturer's instructions (Illumina). Detailed information about data analysis is in the Supplemental Material.

\section{ChIP-seq and DNase sequencing (DNase-seq)}

ChIP was performed as described in Liang et al. (2013). DNase-seq libraries were processed as described previously (Song and Crawford 2010) by Dr. Greg Crawford's laboratory at Duke University. Detailed information about data analysis, SE identification, peak overlap, feature enrichments, and motif analysis is in the Supplemental Material.

\section{Immunofluorescence (IF) and DNA-FISH}

IF and DNA-FISH were carried out as in Jacinto et al. (2015). BACs are described in Supplemental Table S1 and were processed using the DIG-Nick translation mix (Roche). Probes and genomic DNA were denatured simultaneously for $3 \mathrm{~min}$ at $80^{\circ} \mathrm{C}$. The antibodies used were anti-Lamin A (Sigma), anti-Nup153 (SAl mouse ascites from Dr. B. Burke), and antiDIG (Roche). Images were acquired in a Carl Zeiss 710 confocal microscope. For the three-zone scoring, we determined the relative radial positions of FISH spots by calculating the euclidean distance of the FISH spot to the nuclear periphery in the middle plane of the nucleus binned into three concentric zones of equivalent area. Measurements were carried out using Imaris software.

\section{Accession number}

The Gene Expression Omnibus accession number for the data reported in this study is GSE87831. 


\section{Acknowledgments}

We thank Greg Crawford and the Duke University Sequencing Core for generating the DNase I hypersensitivity profiles in U2OS cells, Bas Van Steensel for the plasmid containing the Dam, and the members of the Hetzer laboratory for critical reading of the manuscript. A.I. was supported by a Home Frontier Science Program fellowship. M.W.H. was supported by National Institutes of Health grants R01GM098749, National Institutes of Health Transformative Research Award R01NS096786, the Glenn Aging Foundation, the NOMIS Foundation, the Keck Foundation, and American Cancer Society Award number P30CA014195. M.W.H. and A.I. designed the experiments and wrote the paper. A.I., S.T., and J.C. conducted the experiments. C.B. and A.I. analyzed the data.

\section{References}

Aranda S, Mas G, Di Croce L. 2015. Regulation of gene transcription by Polycomb proteins. Sci Adv 1: e1500737.

Brickner DG, Brickner JH. 2010. Cdk phosphorylation of a nucleoporin controls localization of active genes through the cell cycle. Mol Biol Cell 21: 3421-3432.

Brown CR, Kennedy CJ, Delmar VA, Forbes DJ, Silver PA. 2008. Global histone acetylation induces functional genomic reorganization at mammalian nuclear pore complexes. Genes Dev 22: 627-639.

Brunner M, Millon-Frémillon A, Chevalier G, Nakchbandi IA, Mosher D, Block MR, Albigès-Rizo C, Bouvard D. 2011. Osteoblast mineralization requires $\beta 1$ integrin/ICAP-1-dependent fibronectin deposition. J Cell Biol 194: 307-322.

Buchwalter AL, Liang Y, Hetzer MW. 2014. Nup50 is required for cell differentiation and exhibits transcription-dependent dynamics. Mol Biol Cell 25: 2472-2484.

Capelson M, Hetzer MW. 2009. The role of nuclear pores in gene regulation, development and disease. EMBO Rep 10: 697-705.

Capelson M, Liang Y, Schulte R, Mair W, Wagner U, Hetzer MW. 2010. Chromatin-bound nuclear pore components regulate gene expression in higher eukaryotes. Cell 140: 372-383.

Cavalli G, Misteli T. 2013. Functional implications of genome topology. Nat Struct Mol Biol 20: 290-299.

D'Angelo MA, Gomez-Cavazos JS, Mei A, Lackner DH, Hetzer MW. 2012. A change in nuclear pore complex composition regulates cell differentiation. Dev Cell 22: 446-458.

D'Angelo MA, Hetzer MW. 2008. Structure, dynamics and function of nuclear pore complexes. Trends Cell Biol 18: 456-466.

Dowen JM, Fan ZP, Hnisz D, Ren G, Abraham BJ, Zhang LN, Weintraub AS, Schuijers J, Lee TI, Zhao K, et al. 2014. Control of cell identity genes occurs in insulated neighborhoods in mammalian chromosomes. Cell 159: 374-387.

Griffis ER, Craige B, Dimaano C, Ullman KS, Powers MA. 2004. Distinct functional domains within nucleoporins Nup153 and Nup98 mediate transcription-dependent mobility. Mol Biol Cell 15: 1991-2002.

Guelen L, Pagie L, Brasset E, Meuleman W, Faza MB, Talhout W, Eussen $\mathrm{BH}$, de Klein A, Wessels L, de Laat W, et al. 2008. Domain organization of human chromosomes revealed by mapping of nuclear lamina interactions. Nature 453: 948-951.

Heinz S, Romanoski CE, Benner C, Glass CK. 2015. The selection and function of cell type-specific enhancers. Nat Rev Mol Cell Biol 16: 144-154.

Hnisz D, Abraham BJ, Lee TI, Lau A, Saint-André V, Sigova AA, Hoke HA, Young RA. 2013. Super-enhancers in the control of cell identity and disease. Cell 155: 934-947.

Hyytiäinen M, Keski-Oja J. 2003. Latent TGF- $\beta$ binding protein LTBP-2 decreases fibroblast adhesion to fibronectin. J Cell Biol 163: 1363-1374.

Ibarra A, Hetzer MW. 2015. Nuclear pore proteins and the control of genome functions. Genes Dev 29: 337-349.

Ishii K, Arib G, Lin C, Van Houwe G, Laemmli UK. 2002. Chromatin boundaries in budding yeast: the nuclear pore connection. Cell 109: $551-562$.
Jacinto FV, Benner C, Hetzer MW. 2015. The nucleoporin Nup153 regulates embryonic stem cell pluripotency through gene silencing. Genes Dev 29: 1224-1238.

Kalverda B, Pickersgill H, Shloma VV, Fornerod M. 2010. Nucleoporins directly stimulate expression of developmental and cell-cycle genes inside the nucleoplasm. Cell 140: 360-371.

Kasper LH, Brindle PK, Schnabel CA, Pritchard CE, Cleary ML, van Deursen JM. 1999. CREB binding protein interacts with nucleoporinspecific FG repeats that activate transcription and mediate NUP98HOXA9 oncogenicity. Mol Cell Biol 19: 764-776.

Koziel L, Kunath M, Kelly OG, Vortkamp A. 2004. Ext1-dependent heparan sulfate regulates the range of Ihh signaling during endochondral ossification. Dev Cell 6: 801-813.

Liang Y, Franks TM, Marchetto MC, Gage FH, Hetzer MW. 2013. Dynamic association of NUP98 with the human genome. PLoS Genet 9: e1003308.

Marini B, Kertesz-Farkas A, Ali H, Lucic B, Lisek K, Manganaro L, Pongor S, Luzzati R, Recchia A, Mavilio F, et al. 2015. Nuclear architecture dictates HIV-1 integration site selection. Nature 521: 227-231.

Mekhail K, Moazed D. 2010. The nuclear envelope in genome organization, expression and stability. Nat Rev Mol Cell Biol 11: 317-328.

Meuleman W, Peric-Hupkes D, Kind J, Beaudry J-B, Pagie L, Kellis M, Reinders M, Wessels L, van Steensel B. 2013. Constitutive nuclear laminagenome interactions are highly conserved and associated with A/Trich sequence. Genome Res 23: 270-280.

Nofrini V, Di Giacomo D, Mecucci C. 2016. Nucleoporin genes in human diseases. Eur J Hum Genet 24: 1388-1395.

Nojima Y, Mimura T, Morino N, Hamasaki K, Furuya H, Sakai R, Nakamoto T, Yazaki Y, Hirai H. 1996. Tyrosine phosphorylation of p130Cas in cell adhesion and transformation. Hum Cell 9: 169-174.

Parker SCI, Stitzel ML, Taylor DL, Orozco JM, Erdos MR, Akiyama JA, van Bueren KL, Chines PS, Narisu N, NISC Comparative Sequencing Program, et al. 2013. Chromatin stretch enhancer states drive cell-specific gene regulation and harbor human disease risk variants. Proc Natl Acad Sci 110: 17921-17926.

Pott S, Lieb JD. 2015. What are super-enhancers? Nat Genet 47: 8-12.

Rabut G, Doye V, Ellenberg J. 2004. Mapping the dynamic organization of the nuclear pore complex inside single living cells. Nat Cell Biol 6: 1114-1121.

Raices M, D'Angelo MA. 2012. Nuclear pore complex composition: a new regulator of tissue-specific and developmental functions. Nat Rev Mol Cell Biol 13: 687-699.

Solovei I, Wang AS, Thanisch K, Schmidt CS, Krebs S, Zwerger M, Cohen TV, Devys D, Foisner R, Peichl L, et al. 2013. LBR and lamin A/C sequentially tether peripheral heterochromatin and inversely regulate differentiation. Cell 152: 584-598.

Song L, Crawford GE. 2010. DNase-seq: a high-resolution technique for mapping active gene regulatory elements across the genome from mammalian cells. Cold Spring Harb Protoc doi: 10.1101/pdb.prot5384.

Tan-Wong SM, Wijayatilake HD, Proudfoot NJ. 2009. Gene loops function to maintain transcriptional memory through interaction with the nuclear pore complex. Genes Dev 23: 2610-2624.

Towbin BD, González-Aguilera C, Sack R, Gaidatzis D, Kalck V, Meister P, Askjaer P, Gasser SM. 2012. Step-wise methylation of histone H3K9 positions heterochromatin at the nuclear periphery. Cell 150: 934-947.

Vahedi G, Kanno Y, Furumoto Y, Jiang K, Parker SCJ, Erdos MR, Davis SR, Roychoudhuri R, Restifo NP, Gadina M, et al. 2015. Super-enhancers delineate disease-associated regulatory nodes in T cells. Nature 520: $558-562$.

Van de Vosse DW, Wan Y, Wozniak RW, Aitchison JD. 2011. Role of the nuclear envelope in genome organization and gene expression. Wiley Interdiscip Rev Syst Biol Med 3: 147-166.

Vaquerizas JM, Suyama R, Kind J, Miura K, Luscombe NM, Akhtar A. 2010. Nuclear pore proteins nup153 and megator define transcriptionally active regions in the Drosophila genome. PLoS Genet 6: e1000846.

Worman HJ, Ostlund C, Wang Y. 2010. Diseases of the nuclear envelope. Cold Spring Harb Perspect Biol 2: a000760. 


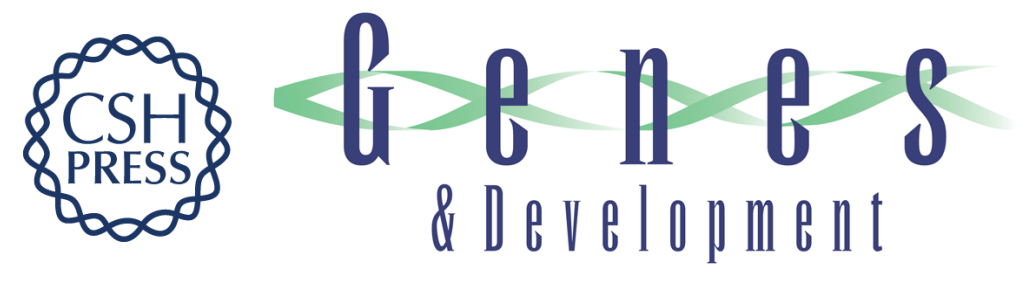

\section{Nucleoporin-mediated regulation of cell identity genes}

Arkaitz Ibarra, Chris Benner, Swati Tyagi, et al.

Genes Dev. 2016, 30: originally published online November 2, 2016

Access the most recent version at doi:10.1101/gad.287417.116

Supplemental

Material

References

Creative

Commons

License

Email Alerting

Service
http://genesdev.cshlp.org/content/suppl/2016/11/02/gad.287417.116.DC1

This article cites 40 articles, 16 of which can be accessed free at: http://genesdev.cshlp.org/content/30/20/2253.full.html\#ref-list-1

This article is distributed exclusively by Cold Spring Harbor Laboratory Press for the first six months after the full-issue publication date (see

http://genesdev.cshlp.org/site/misc/terms.xhtml). After six months, it is available under a Creative Commons License (Attribution-NonCommercial 4.0 International), as described at http://creativecommons.org/licenses/by-nc/4.0/.

Receive free email alerts when new articles cite this article - sign up in the box at the top right corner of the article or click here.

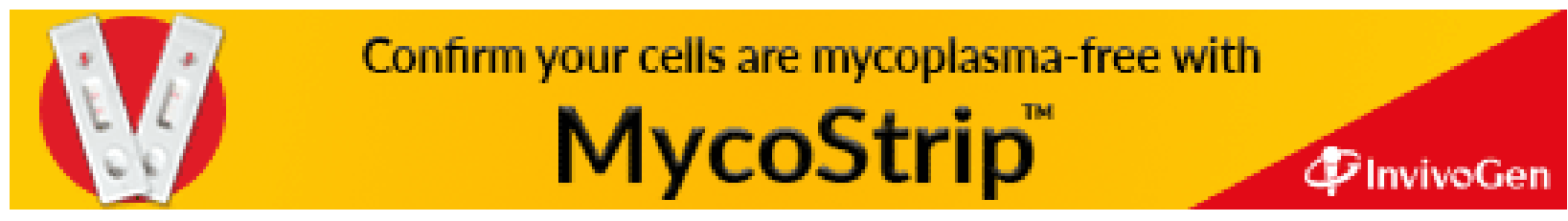

